Duazary / Vol. 16, No. 1 - 2019 / 11 - 13

DOI: http://dx.doi.org/10.21676/2389783X.2546

\title{
EDITORIAL 1
}

\section{La formación de investigadores en el pregrado}

\author{
Guillermo Augusto Ceballos-Ospino ${ }^{1}$ (D), Ubaldo Enrique Rodríguez-de Ávila² (D), \\ Oskarly Pérez-Anaya ${ }^{3}$
}

\begin{abstract}
E algunos contextos se ha cuestionado efectuar investigación en el pregrado, ya que se le considera una labor exclusiva de posgrado; no obstante, es sabido que la mayor parte de los egresados del pregrado no efectuarán investigación como su tarea principal en su accionar laboral, pero si requerirá contar con las herramientas mínimas que le permitan efectuarla cuando le sea requerida o necesaria en su actividad profesional ${ }^{1}$.
\end{abstract}

Debemos tener presente que, en el actual contexto global y local en que vivimos, la educación superior es estratégica para la generación de ciencia, tecnología e innovación (CTI) ya que permiten al nuevo profesional estudiar, comprender y ofrecer soluciones a la problemática del entorno en que se encuentra $^{2-3}$; por ende, para adquirir estas capacidades, es imprescindible aprenderlas para investigar. No se debe olvidar que la investigación vincula la universidad con la sociedad ${ }^{4} \mathrm{y}$, por tanto, la primera está en la obligación de desarrollar estas capacidades de investigación en sus estudiantes y debe estar inmersa en el currículo para su adquisición. Profesores y estudiantes deben poseer una formación básica en metodología de la investigación que permitirá formar profesionales con pensamiento crítico, con deseos de aprendizaje permanente, en búsqueda de plantear problemas y presentar soluciones en su trabajo cotidiano ${ }^{1}$.

Sin embargo, la formación en investigación por lo general se presenta de manera fragmentada y no se lleva a su aplicación inmediata sino se pretende que los estudiantes en el futuro, con el curso de maestrías y doctorados, lleven a cabo su aplicación ${ }^{5}$. Esto implica que el pensamiento crítico, comprensión lectora y demás habilidades necesarias para efectuar investigación sean postergados. Por lo general se asume que investigar se enseña con clases teóricas y no prácticas, más se debe tener en cuenta si los docentes tienen experiencia en investigación y cuentan con las condiciones requeridas por la universidad en que se desempeña. Se debe recordar que la cultura investigativa se debe consolidar fomentando actitudes hacia la misma, bajo la consigna didáctica de aprender haciendo y de esta forma lograr seres humanos integrales y promotores de conocimiento ${ }^{1,5}$.

Para ello se requiere de varios años de adiestramiento, que ayudarán a que cuando se estudie un posgrado, pueda continuar su productividad iniciada en el pregrado ${ }^{6}$. Existen evidencias que seña-

1. Universidad del Magdalena. Santa Marta, Colombia. Correo: guillermoceballos@gmail.com - http://orcid.org/0000-0002-1568-7058

2.UniversidadeFederal do Rio Grande do Norte.Natal,Brasil.Correo:rodriguez.ubaldo@gmail.com-http://orcid.org/0000-0001-5907-001X

3. Universidad del Magdalena. Santa Marta, Colombia. Correo: oskperez123@gmail.com - http://orcid.org/0000-0002-0701-7847 
lan que la investigación en el pregrado logra que estudiantes en este nivel puedan no solo incorporarse a un proyecto sino publicar resultados en revistas científicas acompañados de docentes investigadores, y de esta manera acortan el tiempo para ser productivos en esta actividad ${ }^{7-10}$.

Un problema detectado en la formación de investigadores en el pregrado es el hecho de que, tanto estudiantes como un buen número de docentes, asocian únicamente al trabajo de grado como requisito; como factor agravante está la falta de publicación de la mayoría de estos trabajos, además de que los y estudiantes terminan eligiendo cualquier otra modalidad de grado que la de hacer un proyecto de investigación ${ }^{5,11}$. Es importante tener en cuenta que aquellos docentes que durante su formación de pregrado estuvieron vinculados a grupos de investigación y al finalizar accedieron a cursos de posgrados se convierten en investigadores consumados ${ }^{12}$.

Algo que resulta de interés resaltar es que uno de los principales motivos que han hecho que las universidades implementen la investigación en el pregrado, es que ésta se ha convertido en el canal que aboca a los procesos de acreditación y reconocimiento de alta calidad de las universidades. ${ }^{5}$; la normatividad señala que para que una institución de educación superior aspire a ser considerada como universidad debe contemplar un grado de investigación y estar reflejado en el número de publicaciones científicas, su impacto y en la cantidad de grupos, centros y/o institutos de investigación, incluso para obtener el registro calificado ${ }^{13,14}$.

Es así como la universidad ha implementado como una alternativa a la investigación la creación de "Semilleros de Investigación", opción que ha aportado indicadores que le permiten lograr la excelencia en investigación e innovación ${ }^{14}$. Existe evidencia que los egresados que partici- paron en actividades de semilleros de investigación en el pregrado acceden más fácilmente a maestrías investigativas ${ }^{2}$, publicaron en revistas científicas desde el pregrado, participaron en Congresos con ponencias ${ }^{15,16}$. Por ello, muchas universidades han implementado el programa de semilleros de investigación para identificar y confirmar la vocación investigativa de los estudiantes y docentes que acuden voluntariamente, y en tiempo extracurricular al mismo, bajo la dirección de un investigador de carrera ${ }^{1,17-19}$.

No es menos cierto que el programa de "Jóvenes investigadores e innovadores" de COLCIENCIAS implementado en los años 90s del siglo pasado, dio un impulso al programa de semilleros, quienes egresados del programa de semilleros y de su profesión eran quienes optaban en mayor proporción a dicho programa ${ }^{14}$.

\section{REFERENCIAS BIBLIOGRÁFICAS}

1. Miyahira J. La investigación formativa y la formación para la investigación en el pregrado. Rev Med Hered. 2009; 20 (3): 119-22.

2. Guerrero M. Formación de habilidades para la investigación desde el pregrado. Act.Colom. Psicol. 2007; 10 (2): 190-92.

3. Banco Mundial. Construyendo sociedades del conocimiento: Nuevos retos para la educación terciaria. Banco Mundial. Washington DC: Banco Mundial; 2003.

4. González J, Galindo N, Galindo JL, Gold M. Los paradigmas de la calidad educativa. De la autoevaluación a la acreditación. México. Unión de Universidades de América Latina. 2004.

5. Aldana G. La formación investigativa: su pertinencia en pregrado. Revista Virtual Universidad Católica del Norte. 2012; 35: 367-79. 
6. Rodriguez C, Gijon G. Evolución de la formación de investigadores en pregrado en la Facultad de Medicina, UNAM. Rev Fac Med UNAM. 2001; 44(4): 161-63.

7. Leigthon F, Orrego H, Vargas L. Introducción práctica del estudiante de medicina a la investigación Biomédica. Educ Med Salud. 1981; 15(3): 219-31.

8. Rodríguez Paz CA, Carreón Bringas RM, Ortega Salgado. La investigación en pregrado en la Facultad de Medicina de la UNAM. Gac Med Mex. 1994; 130(Supl 2): 57.

9. Rosales SA, Valverde O. La formación para la investigación en el perfil de carreras de estomatologíade universidadeslatinoamericanas. Rev Cubana Estomatol. 2008; 45:3-4.

10. Guerrero-Useda ME. Formación para la investigación en el contexto universitario. Bogotá: Editorial Universidad Católica de Colombia; 2007.

11. Aldana GM. Características de los egresados del programa de Mercadeo, Publicidad y Ventas de la Fundación Universitaria del Área Andina. Teoría y Praxis Investigativa. 2006; 1(2).

12. Moreno A. Dos pistas para el análisis de los procesos de formación de investigadores en las universidades colombianas. Nómadas. 1997; 7:38-48.
13. Ministerio de Educación Nacional. (1992). Ley 30. Colombia. Ministerio de Educación Nacional. (2010). Decreto $N^{\circ} 1295$.

14. Quintero-CorzoJ, Múnevar-Molina RA, MúnevarQuintero FI. Semilleros de investigación: una estrategia para la formación de investigadores. Educación y Educadores. 2008; 11(1):31-42.

15. Rodríguez C, Gijón G. Evolución de la formación de investigadores en pregrado en la Facultad de Medicina, UNAM. Rev Fac Med UNAM. 2001; 44(4): 161-63.

16. Rodríguez Paz CA, Gijón E. Investigación durante la licenciatura. Rev Fac Med UNAM. 2000; 43(3): 87-9.

17. Guerrero ME. Formación de habilidades para la investigación desde el pregrado. Acta Colombiana de Psicología. 2007; 10(2): 190-92.

18. Torres JM, Maya NA, Capacho LP, Duarte K. Diferencias de las prácticas sexuales entre hombres y mujeres, en estudiantes universitarios durante el primer semestre de 2013. Duazary. 2013;10(2):112-8.19.

19. Tosic M, Narváez-Flórez S, Parra J. Selection of parameters in the design of beach coastal water quality monitoring programs. Intropica. 2013; 8: 43 -51. Disponible en: http://revistas. unimagdalena.edu.co/index.php/intropica/ article/view/731 